\title{
Síndrome de Aicardi: reporte de caso. Más allá de la oftalmología
}

\section{Aicardi syndrome: a case report. Beyond ophthalmology}

\author{
Hae Jin Kim", Rodrigo I. Lozano-Garza², Ulises de Dios-Cuadras ${ }^{1}$, Jesús Guerrero-Becerri2, \\ Andrés Urióstegui-Rojas ${ }^{4}$, Oscar F. Chacón-Camacho ${ }^{5}$, Federico Graue-Wiechers ${ }^{1}$, \\ María E. Hofmann-Blancas ${ }^{2}$ y Juan C. Zenteno-Ruiz ${ }^{5}$
}

${ }^{1}$ Departamento de Retina y Vítreo; ${ }^{2}$ Departamento de Glaucoma; ${ }^{3}$ Departamento de Córnea; ${ }^{4}$ Departamento de Oftalmología Integral; ${ }^{5}$ Departamento de Genética. Instituto de Oftalmología Fundación Conde de Valenciana, Ciudad de México, México

\section{Resumen}

Objetivo: El síndrome de Aicardi fue descrito en 1965 como una tríada de anormalidades que incluye agenesia parcial o total del cuerpo calloso, lagunas coriorretinianas y espasmos infantiles. Se presenta el caso de una paciente con diagnóstico de síndrome de Aicardi que debutó con manifestaciones neurológicas, por lo que se decidió su abordaje multidisciplinario. Caso clínico: Paciente de sexo femenino, de 1 mes de edad, que llega al departamento de genética con antecedentes de epilepsia (espasmos infantiles), agenesia total de cuerpo calloso y disgenesia vertebral con escoliosis encontradas durante la evaluación neurológica previa. El examen oftalmológico reveló microftalmia en el ojo derecho, microcórnea en el ojo izquierdo presentó y resto del segmento anterior sin alteraciones. En la fundoscopia se observaron lesiones coriorretinianas compatibles con lagunas coriorretinianas, así como coloboma de nervio óptico, por lo que se diagnostica síndrome de Aicardi. Conclusiones: Enfatizamos la importancia del examen oftalmológico completo en todos los pacientes para detectar de manera oportuna cualquier anormalidad, además de realizar siempre un abordaje sistémico que permita un diagnóstico sindromático, e individualizar el plan terapéutico.

Palabras clave: Agenesia del cuerpo calloso. Lagunas coriorretinianas. Síndrome de Aicardi.

\section{Abstract}

Purpose: Aicardi syndrome was originally described in 1965, as a triad of abnormalities that includes partial or total agenesis of the corpus callosum, chorioretinal lacunae, and infantile spasms. Case report: A 1-month-old female was evaluated in the Genetics department with a history of epilepsy, total agenesis of the corpus callosum, and vertebral dysgenesis with scoliosis identified during a previous neurological evaluation. An ophthalmological examination revealed microphthalmia of the right eye and microcornea in the left eye; the rest of the anterior segment showed no alterations. Fundoscopy revealed chorioretinal lesions compatible with chorioretinal lacunae that integrated the diagnosis of Aicardi syndrome. Conclusions: We emphasize the importance of a careful ophthalmologic examination in all patients to identify ophthalmological and systemic abnormalities at an early age and the development and implementation of an individualized therapy plan.

Key words: Agenesis of the corpus callosum. Chorioretinal lacunae. Aicardi syndrome.

\section{Correspondencia:}

*Hae Jin Kim

Chimalpopoca, 14

Col. Obrera, Alcaldía Cuauhtémoc

Fecha de recepción: 28-01-2020

Fecha de aceptación: 22-07-2020

C.P. 06800, Ciudad de México, México

E-mail: hjkim.med@gmail.com

0187-4519/@ 2020 Sociedad Mexicana de Oftalmología. Publicado por Permanyer. Este es un artículo open access bajo la licencia CC BY-NC-ND (http://creativecommons.org/licenses/by-nc-nd/4.0/).
Disponible en internet: 12-01-2021 Rev Mex Oftalmol. 2021;95(4):167-170

www.rmo.com.mx 


\section{Introducción}

El síndrome de Aicardi (SA) fue descrito en 1965 por Jean Aicardi y se caracteriza por una tríada de anormalidades que incluye agenesia total o parcial del cuerpo calloso, lagunas coriorretinianas y espasmos infantiles ${ }^{1,2}$.

EI SA se estima que tiene una incidencia de 1:100,000 en los Estados Unidos de América y de 1:93,000 en Holanda. Hasta el momento solo se han descrito 4,000 casos en la literatura médica mundial ${ }^{3,4}$.

El diagnóstico de SA sigue basándose en la presencia de la tríada clínica; sin embargo, los nuevos estudios de imagen permiten diagnosticar casos atípicos 0 incompletos. Los espasmos infantiles son el tipo de epilepsia más común del SA y la mayoría de las veces aparecen en edades tempranas (3-4 meses) $)^{1,3}$.

Una característica distintiva del SA, además de los hallazgos cerebrales, son las manifestaciones oftalmológicas. Las lagunas coriorretinianas son patognomónicas del SA. Otras alteraciones oculares que se pueden encontrar son microftalmia, persistencia de la membrana iridopupilar, coloboma coriorretiniano, neovascularización coroidea, anormalidades del nervio óptico incluyendo hipoplasia, coloboma, anomalía de Morning Glory, desprendimiento de retina seroso y extensión de tejido glial al disco óptico ${ }^{5-9}$.

Entre las alteraciones no neurológicas se encuentran anormalidades vertebrales, anormalidades costales, como ausencia o bifurcación de las costillas, y escoliosis, que se presentan en la mitad de los $\operatorname{casos}^{1,3}$.

La importancia del presente reporte de caso radica en que se trata de una paciente que inició con manifestaciones neurológicas, por lo cual se decidió su valoración por los servicios de genética y oftalmología.

El objetivo es dar a conocer a la comunidad oftalmológica mexicana la importancia de un abordaje interdisciplinario y multidisciplinario para los síndromes genéticos raros que manifiestan signos oftalmológicos clave y permiten un diagnóstico sindrómico certero.

\section{Caso clínico}

Paciente de sexo femenino de 1 mes de edad que se presenta al departamento de genética con antecedentes de epilepsia, agenesia total del cuerpo calloso y disgenesia vertebral con escoliosis, los cuales fueron identificados durante la evaluación neurológica previa (Fig. 1). Fue producto de tercera gesta de padres no consanguíneos y nació por cesárea con embarazo de término sin complicaciones. No requirió maniobras de reanimación cardiopulmonar avanzada, el peso al nacer fue de $2,900 \mathrm{~g}$

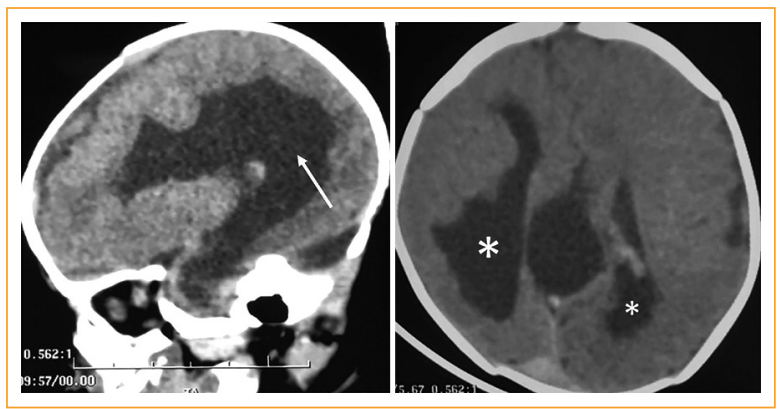

Figura 1. Imágenes de tomografía que muestran agenesia total de cuerpo calloso (flecha) y heterotopia cortical (asteriscos).

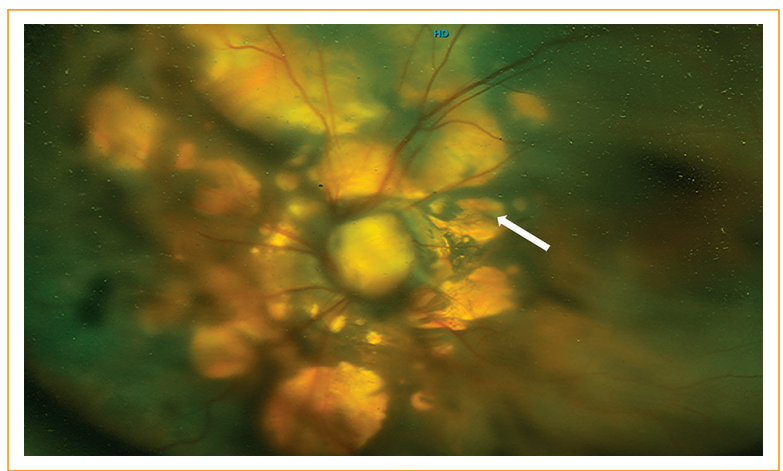

Figura 2. Fundoscopia del ojo izquierdo que muestra coloboma del nervio óptico y múltiples lesiones planas coriorretinianas de distribución peripapilar con pigmento variable en sus bordes, que son sugestivas de lagunas coriorretinianas (flecha).

y la talla fue de $49 \mathrm{~cm}$; se desconocen las puntuaciones en las escalas de Apgar y Silverman-Anderson.

En la exploración física al mes de edad, la paciente presentaba hipotonía generalizada sin organomegalias. En el examen oftalmológico mostró acortamiento de los fondos de saco de ambos ojos, y en el ojo derecho microftalmia, con el resto de las estructuras no valorables. En el ojo izquierdo se halló microcórnea y el resto del segmento anterior sin alteraciones. La fundoscopia del ojo izquierdo mostró el nervio óptico con excavación asimétrica con un remanente escaso nasal, pigmento peripapilar mínimo y vasculatura anormal con emergencia central que corresponde a un coloboma. Además, presenta múltiples lesiones coriorretinianas de aproximadamente un área de disco, de coloración amarillenta, ovoideas, con pigmento variable en sus bordes y distribuidas en el área peripapilar confinadas en el polo posterior, las cuales son sugestivas de lagunas coriorretinianas (Fig. 2). En la ecografía ocular se observó una longitud 
Tabla 1. Criterios diagnósticos modificados para el síndrome de Aicardi

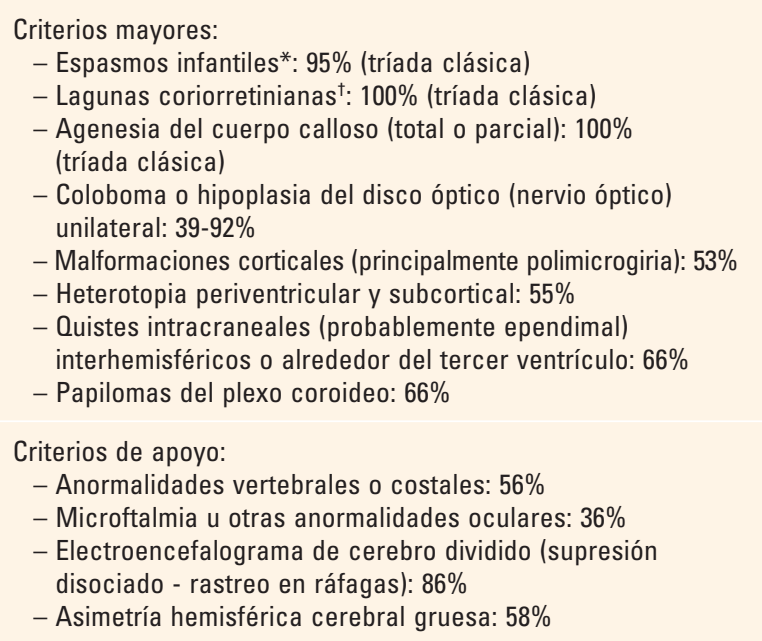

*Puede ser reemplazado por otros tipos de epilepsia (usualmente focal).

tPresente (o probablemente presente) en todos los casos.

axial del ojo derecho de $14.94 \mathrm{~mm}$, con lo que confirmamos el diagnóstico de microftalmia. Como parte del abordaje, el departamento de genética solicitó cariotipo, que reportó 46, XX. El departamento de neurología indicó manejo terapéutico con ácido valproico $(250 \mathrm{mg} / 5 \mathrm{ml})$ cada 8 horas por vía enteral, clonazepam $(0.48 \mathrm{mg})$ cada 24 horas por vía oral, vigabatrina $(500 \mathrm{mg})$ cada 12 horas por vía oral y levetiracetam $(100 \mathrm{mg} / 2.2 \mathrm{ml})$ cada 12 horas por vía intravascular. El departamento de oftalmología integral indicó valoración por parte de los servicios de glaucoma, retina, oculoplástica y visión baja del Instituto de Oftalmología, que decidieron un abordaje en conjunto para ambliopía de origen mixto y elaboración de prótesis ocular para el ojo derecho, así como explicación del pronóstico visual reservado para ambos ojos por la patología de base.

Desafortunadamente, la paciente falleció por complicaciones de una neumonía adquirida en la comunidad que cursó con síndrome de dificultad respiratoria, choque séptico y finalmente paro cardiorrespiratorio con 1 año y 7 meses de edad.

\section{Discusión}

Para establecer el diagnóstico de SA se necesita la presencia de la tríada clásica o la presencia de dos componentes de la tríada clásica y al menos dos criterios mayores o de soporte ${ }^{1,3}$ (Tabla 1). Nuestra paciente presentó la tríada clásica, y además dos criterios de apoyo, por lo cual se llegó al diagnóstico de SA.
El examen oftalmológico es clave en el diagnóstico y debe ser realizado por un oftalmólogo con entrenamiento en oftalmología pediátrica o por un genetista con alta especialidad en padecimientos oftalmológi$\cos ^{7}$. Dentro de los casos reportados en la literatura destaca el hallazgo de lagunas coriorretinianas hasta en el $100 \%$ de los casos, y son consideradas patognomónicas en el SA ${ }^{5-9}$; se caracterizan por ser lesiones amarillas 0 blanco-amarillentas, redondeadas, de tamaño variable y de localización peripapilar en el polo posterior. Histopatológicamente corresponden a epitelio pigmentado retiniano hipopigmentado con desorganización de la retina. Las anomalías del nervio óptico son frecuentes y los colobomas son la presentación más común, hasta en el $39 \%$ de los casos. Sin embargo, también se han descrito otras alteraciones, ocupando el segundo lugar la anomalía de Morning Glory, en el $21-86 \%$ de los casos. También se han descrito la presencia de microftalmia, más habitual del lado derecho, y de otras alteraciones tales como anormalidades de los vasos retinianos ${ }^{5-9}$.

Aunque los genes causantes de SA no han sido reconocidos hasta la fecha, se sospecha que el origen es una mutación genética ligada al $\mathrm{X}$, ya que los casos reportados son exclusivos en pacientes de sexo femenino, tal como nuestro caso. Los pocos casos en varones fueron con cariotipo 47, XXY y uno con 46, XY. Algunos autores sugieren que el proceso de inactivación del cromosoma $\mathrm{X}$ puede explicar la variabilidad $\mathrm{y}$ la asimetría observadas en la enfermedad, y esto se ve respaldado por una prevalencia más alta de lo normal de inactivación cromosómica $X$ sesgada en los linfocitos periféricos en el SA, sobre todo en aquellos pacientes con involucro neurológico ${ }^{10}$.

El neurólogo pediatra con experiencia en el manejo de espasmos infantiles es esencial para el tratamiento a largo plazo de los pacientes con SA, quienes con frecuencia requieren varios antiepilépticos para el control adecuado de las convulsiones, como en nuestro caso. En las series de casos se encuentran como características principales la agenesia total o parcial del cuerpo calloso (100\%) y los espasmos infantiles (95\%), signos que pertenecen a la tríada clásica. Entre otros datos se pueden encontrar heterotopía cortical $(55 \%)$, polimicrogiria $(53 \%)$, quistes intracraneales $(66 \%)$ y papilomas de los plexos coroideos $(66 \%)^{1,4,6}$.

Las intervenciones adicionales incluyen terapia física, terapia ocupacional, terapia del habla y terapia visual, las cuales deben iniciarse tan pronto como sea posible para asegurar el mejor desarrollo y la funcionalidad de los diferentes órganos de los sentidos. Los 
defectos costovertebrales pueden llevar a escoliosis, como en nuestro caso. El adecuado apoyo musculoesquelético y el tratamiento para la prevención de complicaciones relacionadas se encuentran indicados ${ }^{3}$.

La tasa de supervivencia es del $76 \%$ hasta a los 6 años y del $40 \%$ hasta los 14 años de edad. La mayoría de los pacientes tienen deterioro en los primeros 5-6 años de vida y es complicado determinar las complicaciones de las malformaciones y las consecuencias de una epilepsia no controlada. La mayoría de los pacientes permanecen con estatura baja como consecuencia de la escoliosis'.

Como limitaciones del presente caso tenemos el pobre seguimiento de la paciente por parte del departamento de oftalmología, debido a numerosos ingresos al servicio de urgencias pediátricas por cuadros repetitivos de infección de vías respiratorias bajas, donde no solicitaron interconsultas de seguimiento. Esto llevo a dificultades en el seguimiento externo de la paciente. Finalmente, no se pudo valorar su evolución debido a que la paciente falleció con 1 año y 7 meses de edad.

En conclusión, debemos enfatizar que es necesario el examen oftalmológico completo y que este permite individualizar el plan terapéutico. En cuanto a la terapia visual, recomendamos una implantación temprana de prótesis en casos de acortamiento de los fondos de saco, y lograr un aspecto estético y anatómico adecuado. En caso de que exista integridad de las vías visuales, hay que realizar estímulo visual (apoyándonos de oftalmólogos con alta especialidad en visión baja) y consejo psicológico para el paciente y los familiares.

\section{Financiamiento}

Los autores no recibieron ningún financiamiento para la realización de este artículo.

\section{Conflicto de intereses}

Los autores declaran no tener ningún conflicto de intereses.

\section{Responsabilidades éticas}

Protección de personas y animales. Los autores declaran que para esta investigación no se han realizado experimentos en seres humanos ni en animales.

Confidencialidad de los datos. Los autores declaran que han seguido los protocolos de su centro de trabajo sobre la publicación de datos de pacientes.

Derecho a la privacidad y consentimiento informado. Los autores han obtenido el consentimiento informado de los pacientes y/o sujetos referidos en el artículo. Este documento obra en poder del autor de correspondencia.

\section{Bibliografía}

1. Aicardi J. Aicardi syndrome. Brain Dev. 2005;27:164-71.

2. Fernández-Ramos JA, López-Laso E, Simón-De Las Heras R, Camino-León R, Guerra-García P, Camacho-Salas A, et al. Aicardi syndrome: retrospective study of a series of seven case reports. Rev Neurol. 2013;57:481-8.

3. Sutton VR, Hopkins BJ, Eble TN, Gambhir N, Lewis RA, Van den Veyver IB. Facial and physical features of Aicardi syndrome: infants to teenagers. Am J Med Genet A. 2005;138A:254-8. .

4. Kroner BL, Preiss LR, Ardini MA, Gaillard WD. New incidence, prevalence, and survival of Aicardi syndrome from 408 cases. J Child Neurol. 2008;23:531-5.

5. Fruhman G, Eble TN, Gambhir N, Sutton VR, Van den Veyver IB, Lewis RA. Ophthalmologic findings in Aicardi syndrome. J AAPOS. 2012;16:238-41.

6. Cabrera MT, Winn BJ, Porco T, Strominger Z, Barkovich AJ, Hoyt CS, et al. Laterality of brain and ocular lesions in Aicardi syndrome. Pediatr Neurol. 2011;45:149-54.

7. Shah PK, Narendran V, Kalpana N. Aicardi syndrome: the importance of an ophthalmologist in its diagnosis. Indian J Ophthalmol. 2009:57:234.

8. Martel JN, Rutar T, Lujan BJ, de Alba Campomanes A. (2011). Chorioretinal architecture in Aicardi syndrome: an optical coherence tomography and fluorescein angiography study. J AAPOS. 2011;15:308-10.

9. Galdós M, Martínez R, Prats JM. Clinical outcome of distinct Aicardi syndrome phenotypes. Arch Soc Esp Oftalmol. 2008;83:29-36.

10. Lund $C$, Striano $P$, Sorte HS, Parisi $P$, lacomino $M$, Sheng $Y$, et al. Exome sequencing fails to identify the genetic cause of Aicardi syndrome. Mol Syndromol. 2016;7:234-8. 